\title{
Forcing of the Martian polar annulus by Hadley cell transport and latent heating
}

\author{
R. K. Scott ${ }^{1 *}$, W. J. M. Seviour ${ }^{2}$, D. W. Waugh ${ }^{3}$ \\ ${ }^{1}$ School of Mathematics and Statistics, University of St Andrews, St Andrews, UK \\ ${ }^{2}$ Cabot Institute, School of Geographical Sciences, University of Bristol, Bristol, UK
}

Department of Earth and Planetary Sciences, The Johns Hopkins University, Baltimore, Maryland, USA

${ }^{*}$ Correspondence to: R. K. Scott, School of Mathematics and Statistics, University of St Andrews, North Haugh, St Andrews, KY16 9SS, UK. Email: rks4@st-andrews.ac.uk

A hierarchy of idealized models is used to investigate the roles of Hadley cell forcing and latent heat release from carbon dioxide condensation in determining the annular potential vorticity structure of the Martian winter polar vortex. The angular momentum conserving Hadley cell model of Lindzen and Hou with summer hemisphere heating maximum of appropriate strength and latitude produces a strong westerly jet near $60 \mathrm{~N}$, similar in strength to the winter polar night jet on Mars. Although the corresponding potential vorticity profile in the angular momentum conserving and thermal wind regions has no annular structure resembling the Martian one, there is an implied deltafunction at the discontinuity in zonal wind. This delta-function is smoothed out by explicit diffusion in full axisymmetric model integrations forming a partial annular structure, though a local maximum in potential vorticity at the pole persists and is further enhanced when cooling representing the polar night is included. A distinct a polar minimum and clear annular potential vorticity structure is obtained, however, when an additional representation of polar latent heating is also included. Full eddy-permitting shallow water model integrations confirm the basic structure obtained by the axisymmetric model and suggest a nominal value of viscosity appropriate as a representation of the effects of eddy mixing. Instability of the polar annulus leads to vacillation type behaviour involving eddy growth and annulus disruption, followed by reformation under the influence of radiative relaxation. The degree of transience and mean eddy activity both show an increase with stronger latent heating and a resulting deeper polar potential vorticity minimum, showing that mixing in polar regions may be dependent on details of polar carbon dioxide condensation. Vacillation timescales are also shown to vary with radiative timescale, but through a modification of instability growth rate rather than direct radiative restoration. Copyright (C) 0000 Royal Meteorological Society

Key Words: Mars, polar vortex, Hadley cell, potential vorticity, latent heating

Received ...

Citation: ...

This article has been accepted for publication and undergone full peer review but has not been through the copyediting, typesetting, pagination and proofreading process, which may lead to differences between this version and the Version of Record. Please cite this article as doi: $10.1002 /$ qj.3786 


\section{Introduction}

The Martian polar atmosphere in Northern hemisphere winter is characterized by a strong cyclonic zonal mean circulation, the detailed structure of which has been inferred from the temperature retrievals of the Mars Global Surveyor (Banfield et al. 2004; McConnochie 2007) as well as model studies (Barnes and Haberle 1996; Waugh 16) and reanalysis products (Montabone et al. 'eybush et al. 2012; Montabone et al. 2014). TP poitr winds broadly resemble those of the Earth's wh rratospheric polar vortex but with some important diacrences, in particular regarding the potential vorticity on and the forcing mechanisms responsible for creating the circulation (e.g. Mitchell et al. 2015, for a compative overview). Specifically, the Martian polar re $x$ is observed to have an annular structure in potential vorticity which has been proposed to be related to latent heat release from condensation of carbon dioxide in the old temperatures of the polar night (Toigo et al. 20 (7) ince such a structure is expected to be unstable to 1 Prizy tal wave perturbations, the observations raise the questron of how it is sustained on timescales of the seasonal c) icie.

In con rast to the Earth, the Martian atmosphere does not have a distinct stratosphere and accordingly the $\mathrm{M}$ artian jolar vortex may be considered as an essentially tre ospheric feature. It is driven in part by the outflow from a Aadley cell extending from the southern (summer) $\mathrm{h}$ - Inisph re as far as $60^{\circ}$ or $70^{\circ} \mathrm{N}$ and the associated angular moncritum transport within this cell (e.g. Leovy 2001). As -11, it may be more correctly likened to the Earth's stutrnpical jet (Waugh et al. 2016). Because the Martian surface has no oceans and a relatively thin atmosphere, the effective solar heating of the atmosphere is more strongly controlled by the planetary obliquity than on Earth. Consequently there is greater hemispheric asymmetry in the location of maximum heating during the seasonal cycle and greater asymmetry between winter and summer Hadley cell branches. Additionally, large zonal mean topography and, to a lesser extent, orbital eccentricity both contribute to higher effective summer heating in the Southern hemisphere and consequently a stronger and broader Northern hemisphere winter Hadley cell branch than its Southern hemisphere winter counterpart (Richardson and Wilson 2002; Zalucha et al. 2010). For this reason, the Martian polar vortex is significantly stronger and more defined in the Northern hemisphere winter.

Details of the structure of the Martian polar vortex are tied to the nature of the angular momentum within the Hadley cell. Angular momentum is transported up to the edge of the cell but not significantly beyond it, where the zonal flow is closer to a state of thermal wind balance with the radiative equilibrium temperature. Depending on details of the radiative equilibrium over the pole, the potential vorticity resulting from the Hadley cell could in certain circumstances maximize near the vortex edge, accounting for the observered annular structure, which has a minimum in potential vorticity over the pole and a maximum around $70^{\circ} \mathrm{N}$ (Mitchell et al. 2015; Waugh et al. 2016). However, the connection between Hadley cell and polar vortex structure has not been studied in detail. As will be seen further below, details of the potential vorticity distribution over the pole depend on the relative strengths of Hadley cell angular momentum transport and polar diabatic forcing. Diabatic forcing on Mars is not limited to cooling in the winter night, where temperatures are low enough that latent heat release from carbon dioxide condensation may play a significant role in the seasonal mean temperature budget. In particular, a recent modelling study found that when parametrization of carbon dioxide condensation was removed, the polar vortex remained monopolar and did not develop any annular structure (Toigo et al. 2017).

It is well known from theory and numerous modelling studies that annular distributions of potential vorticity can in general be shear unstable (Waugh and Dritschel 1991; Dritschel and Polvani 1992). In the context of the Martian vortex, Seviour et al. (2017) demonstrated, in a spherical 
shallow water model that, in the absence of any external forcing, an annular structure broadly representative of the observered potential vorticity rapidly collapses into a monopolar structure. Seviour et al. (2017) examined both the linear and nonlinear nature of this barotropic instability. Typical vortex geometries were found to be linearly unstable to zonal wavenumbers in the range 3-6, with maximum growth rates on time scales in the range of $1-3$ ols. Consquently, in nonlinear calculations, the ructure was found to persist when the vortex was sy aine by a relaxational forcing to the initial annular stru on comparable time scales, broadly representative of cin raciative relaxational time scale on Mars Eckermann 011). Seviour et al. (2017) found that when reraxation timescales were much longer than the timescale for instablitity the late-time vortex became more monopolar, er eas when relaxation timescales were short the late-time vortex - mained more strongly annular. When instability and relaxational timescales are comparable, the nonlinear

\section{. ions that exist in the statistically stationary state} repalance between zonal forcing and eddy growth $\mathrm{d}$ to in tability. Observations indicate zonal asymmetries that may indeed represent coherent potential vorticity stuctures resulting from the instability of a continuously ced an nulus.

In this paper, we consider the extent to which the simple Hadley ell models of Held and Hou (1980) and Lindzen an How (1988), which have a long history of application in tho $\mathrm{s}$ atmospheric circulation, may provide insight into $\mathrm{h}, \mathrm{w}$ the alance of dynamical forcings controls the structure of the ivlartian polar vortex. Such axisymmetric models have als $\mathrm{ch}$ appied to the study of Martian great dust storms (Soh eider 1983). In addition to examining the purely axisymmetric formation mechanisms, we also consider how the annular potential vorticity distribution persists in the presence of shear instability and how the saturation of nonlinear eddy motions arising from the instability depends on the relative strengths of Hadley cell transport and diabatic forcing. In the nonaxisymmetric calculations, the paper extends the work of Seviour et al. (2017) primarily in that the forcing is a now closer representation of the angular momentum transport on Mars. Specifially, the polar annulus is forced not through a direct relaxation to a prescribed annular structure, but rather through a relaxation to an unrealizable equilibrium state that implies the development of a latitudinally broad Hadley cell.

The paper is structured as follows. In section 2, we review the angular momentum conserving (AMC) models of steady axisymmetric flow and consider the structure of the solutions in the parameter regime relevant for the Martian Northern hemisphere winter solstice. We also introduce simple representations of diabatic cooling and heating within the thermal equilibrium of the polar region. In section 3, we examine the extent to which the $\mathrm{AMC}$ model solutions are realized in an axisymmetric shallow water model with relaxation to a similar thermal equilibrium profile and examine how the inviscid solution is approached with decreasing viscosity. We also consider briefly how this model, for a given viscosity appropriate to nonlinear eddy mixing, may be used to quickly infer the zonal mean circulation under different conditions, varying the planetary obliquity and solar forcing. In section 4 , we consider the competition between dynamical instability and forcing by examining a full eddy resolving shallow water model. The dependence of the eddy structure of the annulus on different forcing parameters is also considered. Section 5 concludes.

\section{The LH88 AMC model}

Held and Hou (1980) and Lindzen and Hou (1988) (hereafter LH88) examined the steady state axisymmetric circulation generated by a solar heating that maximized in the tropics. The symmetric case in which the heating maximum is located exactly on the equator (Held and Hou 1980) can be considered as representing equinox conditions, while the off-equator case (LH88) pertains to solsticial or seasonally evolving conditions and is the relevant model for the present study. In these models, the heating is represented by a relaxation to a radiative-convective equilibrium temperature 


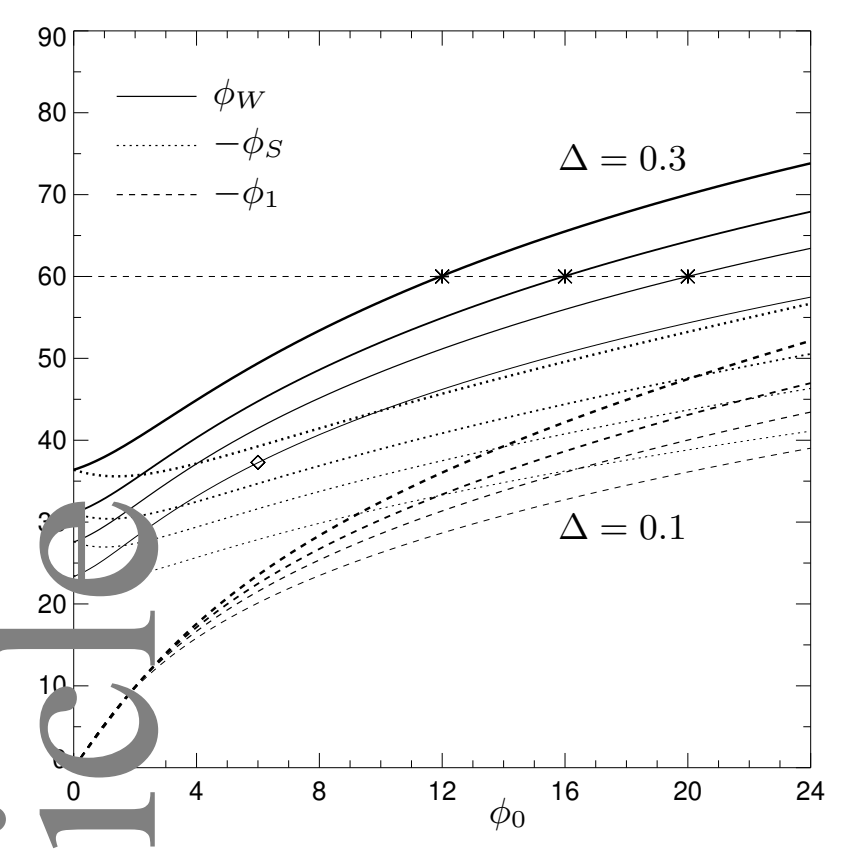

Fi rume 1 ransition latitudes for the AMC solutions of LH88. The values $\Delta=0.1,0.15,0.2,0.3$ are shown with increasing line thickness. Stars in acate cases presented below; the diamond indicates that of LH88 Fig.9c.

$\theta_{e}$ Thodels are based on the result that the zonal flow In th rmal wind balance with such a heating would have a surface wind maximum that exceeds the planetary rotation, which is unrealizable in the absence of additional forcings (1 Hide 1959). The radiative equilibrium state thus cannot be attained in a latitudinal band surrounding the heating maximv $\Lambda$, and the difference between actual temparature $\theta$ ar $d \theta$ in plies the existence of a transverse circulation in the $\mathrm{m}$ al plane. The existence of the transverse circulation ir then absolute angular momentum must be co $\tan \mathrm{t}$ within such a region.

Fr. nodel is made explicit by consideration of the zonal $\mathrm{n}$ oment $\mathrm{m}$ equation, which for steady, axisymmetric flow simplities to

$$
v \frac{d m}{d \mu}=0
$$

wh $m=a \cos \phi(a \Omega \cos \phi+u)$ is the absolute angular momentum, $u$ and $v$ are zonal and meridional components of velocity, $\phi$ is latitude, $\mu=\sin \phi, \Omega$ is the planetary rotation, and $a$ is the planetary radius. Equation (1) can be satisfied by the vanishing of either factor. Thus, inside the angular momentum conserving (AMC) region near the heating maximum, $v \neq 0$ but the flow has constant angular momentum, $d m / d \mu=0$, or equivalently zero absolute vorticity; the associated zonal flow is in thermal balance with a $\theta \neq \theta_{e}$. Outside the AMC region, $v=0$ and the zonal wind is in thermal balance with $\theta_{e}$.

LH88 used a hemispherically asymmetric equilibrium heating with a latitudinal profile of the form

$$
\theta_{e} / \theta_{0}=1+\Delta\left(\left(\frac{1}{3}-\left(\sin \phi-\sin \phi_{0}\right)^{2}\right)\right.
$$

where here $\theta_{e}$ represents an average in the vertical over the depth $H$ of the troposphere. The heating has a maximum at latitude $\phi_{0}$ and horizontal temperature difference $\Delta$. In their model formulation, the flow is assumed to be angular momentum conserving between latitudes $\phi_{s}$ and $\phi_{w}$, the summer and winter hemisphere termini, and in thermal wind balance poleward of these latitudes. The location of maximum ascent, $\phi_{1}$, differs from the location of the maximum heating, $\phi_{0}$, and is such that the summer cell is significantly weaker than the winter cell. Indeed, one of the main conclusions of LH88 was that even weakly asymmetric forcing results in a large asymmetry between the summer and winter cells, to the extent that the annual averaged transport of seasonally varying heating must greatly exceed the transport due to the annually averaged heating.

For a specified asymmetry $\phi_{0}$ and heating strength $\Delta$, the latitudes $\phi_{1}, \phi_{s}$, and $\phi_{w}$ are determined uniquely by matching conditions between the AMC and thermal wind regions, obtained from conservation of energy and continuity of temperature at $\phi_{s}$ and $\phi_{w}$. The matching conditions were given in equations (8)-(11) of LH88 and lead to a system of three nonlinear equations in the unknowns $\phi_{1}, \phi_{s}$, and $\phi_{w}$. For completeness and for ease of future use, we provide the equations in the appendix. The main point, however, is that the solutions are controlled by only two parameters: the asymmetry $\phi_{0}$ and the combination $R=B \Delta$, where $B=g H / a^{2} \Omega^{2}$ is a planetary Burger number. Thus the effects of the heating strength $\Delta$ and physical planetary parameters affect the solution only in the combination $R=B \Delta$, rendering it unnecessary to consider these parameters in isolation. Here 
we use planetary parameters as given in Seviour et al. (2017), essentially $L_{D}=\sqrt{g H} / 2 \Omega=1800 \mathrm{~km}$ with $a=$ $3390 \mathrm{~km}$, giving $B=1.12$.

The AMC model is readily extended beyond the Earth regime to one more relevant to the Martian atmosphere simply by considering larger values of $\phi_{0}$ and/or $\Delta$, which can be increased until the northern edge of the AMC region coimcides approximately with the observed extent of the Had lev 11 on Mars. Figure 1 shows the latitudes $\phi_{1}, \phi_{s}$, and unction of $\phi_{0}$ and for several values of $\Delta=R B$. $\mathrm{T}^{1}$ sym bol $\phi_{0}=6^{\circ}$ on the curve with smallest $\Delta$ indicates the considered in LH88 Figure 9c. For the Martian at nuspmore, a winter terminus at approximately $60^{\circ} \mathrm{N}$ is

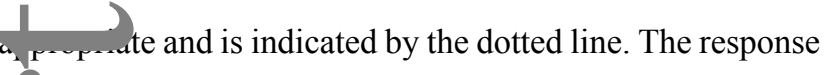
at anv nolnt on this line can be obtained with an appropriate combination of $\phi_{0}$ and $\Delta$; three combinations are indicated al vill be used for further analysis, $\left(\phi_{0}, \Delta\right)=\left(12^{\circ}, 0.3\right)$, $\left(16^{\circ}, 0^{\prime}\right)$, and $\left(20^{\circ}, 0.15\right)$. As would be expected, the more symmetric the forcing (smaller $\phi_{0}$ ) the larger the $\Delta$ required he same maximum latitude of the winter cell.

r. of $\theta / \theta_{0}$, vertically averaged zonal wind $u$, and a sc lute orticity $\zeta_{a}$ for three combinations of $\phi_{0}$ and $R$ with $\phi_{v}=60^{\circ}$ (indicated by the symbols on Fig. 1) are shown in Fig. 2. The change in imposed temperature difference $\Delta$ a be sen in the profiles of $\theta$ and to a lesser extent in those of $u$ and $\zeta_{a}$. Essentially, the parameters controlling the simmer ind winter extents and the location of maximum extyt all vary more or less similarly with $\phi_{0}$; thus fixing $\phi_{\text {roc }} \mathrm{s}$ in similar, but not exactly the same, $\phi_{s}$ and $\phi_{1}$ a a a br adly similar parabolic (in sine latitude) form for $u$. The unierences will be explored more in the eddy resolving mod oclow.

In all three cases, the absolute vorticity is exactly zero within the AMC region (bounded by the thin vertical lines) and equal to a profile in thermal wind balance with $\theta_{e}$ outside. At first sight, there is no suggestion of a nonmonotonic, annular distribution as found in the Martian reanalysis. In fact, the profiles shown all have a clear polar maximum of absolute vorticity, a consequence of the thermal wind balance with the equilibrium state $\theta_{e}$. However, the absolute vorticity profiles shown in Fig. 1 hide one fundamental aspect, namely that, regardless of the details of the polar $\theta_{e}$, the LH88 solution has a discontinuity in the wind profile at $\phi_{w}$, a shear line with an implied $\delta$-function of vorticity. In a viscous model or in a model with resolved eddy mixing, such a shear line would be smoothed out over a finite latitude, with the $\delta$-function broadening but retaining a local maximum. Thus the profiles shown do suggest some form of annular potential vorticity distribution might arise purely as a result of the Hadley cell outflow, but to predict the detailed horizontal structure requires a model in which the transition between $\mathrm{AMC}$ and equilibrium regions is correctly represented. This will be addressed in the following two sections.

Before turning to the full dynamical equations, however, we note that the details of the polar vorticity depend only on the $\theta_{e}$ poleward of $\phi_{w}$. Also, provided $\theta_{e}\left(\phi_{w}\right)$ itself is unchanged, the AMC winds are unaffected by changes to $\theta_{e}$ poleward of $\phi_{w}$, since they are determined purely by matching conditions involving $\theta$ at the transition latitudes. Therefore, by modifying $\theta_{e}$ poleward of $\phi_{w}$ the polar vorticity profile can be changed directly, without altering the AMC circulation and vorticity structure south of $\phi_{w}$.

To illustrate, we consider how the solution changes when $\theta_{e}$ is modified to $\theta_{e}+\theta_{e}^{\prime}$, where

$$
\theta_{e}^{\prime}\left(\phi_{c}, Q\right)=Q \theta_{0} \cos ^{2}\left(\frac{\pi}{2} \frac{\phi-\pi / 2}{\phi_{c}-\pi / 2}\right)
$$

Here, $Q<0$ corresponds to an additional cooling and $Q>0$ to a heating. With $\phi_{c}=\phi_{w}$ the modification is restricted poleward of $\phi_{w}$. The response to the cases $Q=$ \pm 0.1 are shown dotted and dashed in Fig. 1. Cooling the pole (lowering $\theta_{e}$ ) causes the polar maximum in absolute vorticity to increase, as well as the zonal velocity, in accordance with thermal wind balance. Conversely warming causes a decrease in the vorticity over the pole and an increase towards $\phi_{w}$, resulting in a clear nonmonotonic profile and polar minimum, even neglecting the contribution 
(a) $\phi_{0}=12, \Delta=0.3$
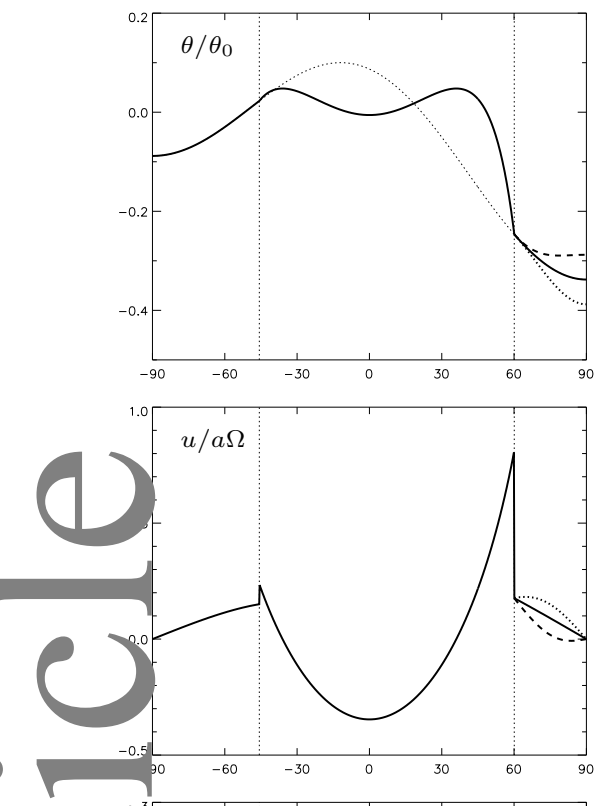

$\zeta_{a}$

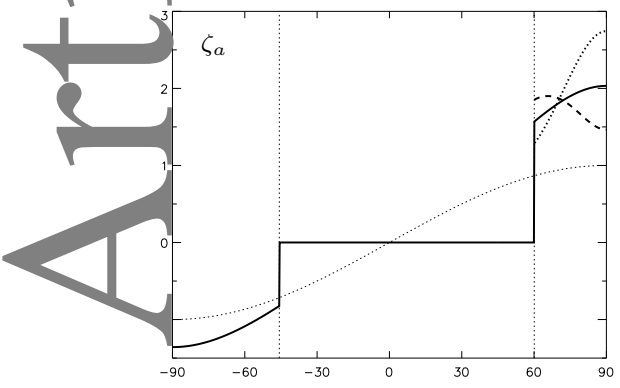

(b) $\phi_{0}=16, \Delta=0.2$
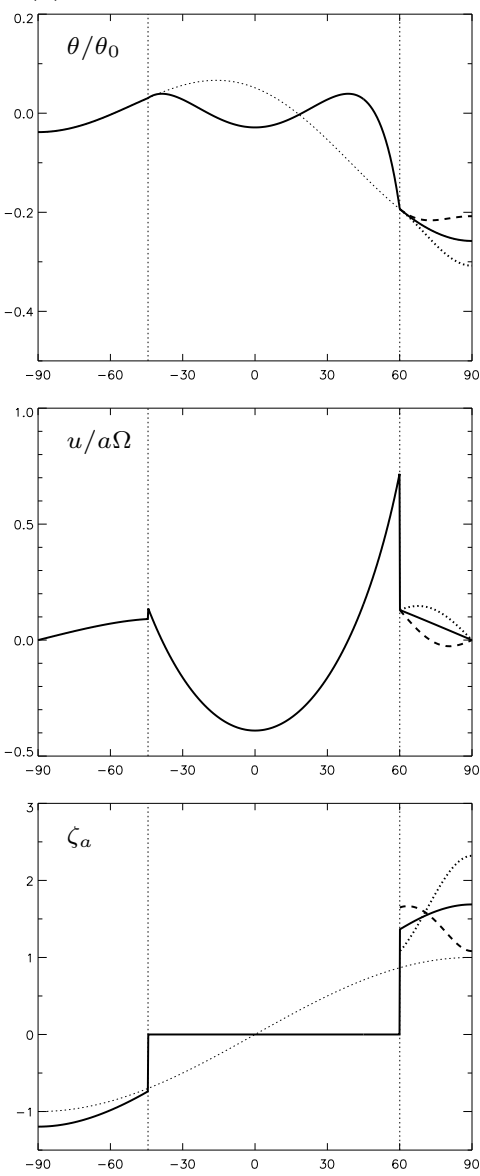

(c) $\phi_{0}=20, \Delta=0.15$
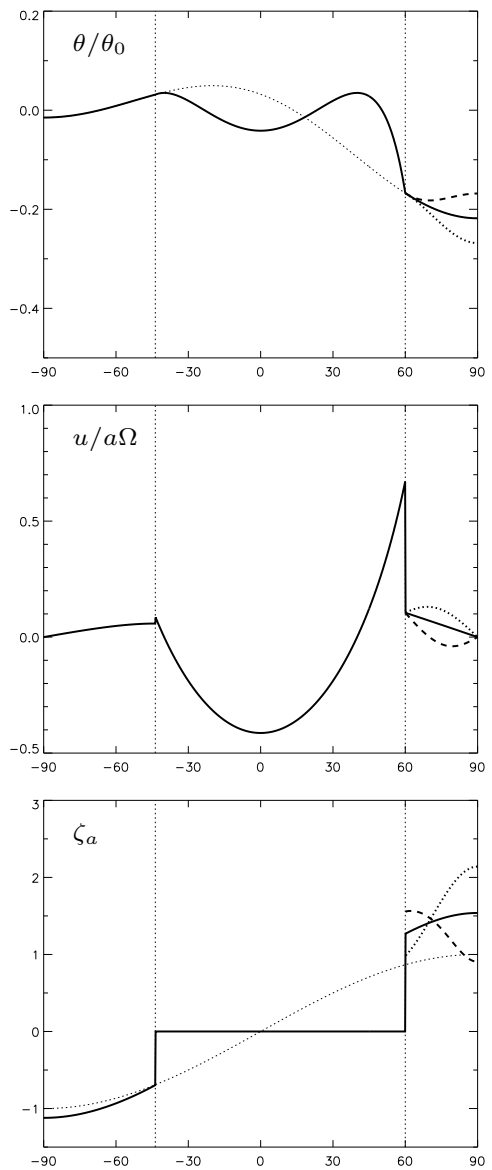

Fiamm ? rofiles of $\theta / \theta_{0}, u$, and $\zeta_{a}$, for solutions of LH88 with $\phi_{w}=60^{\circ}$. Vertical dotted lines indicate $\phi_{w}$ and $\phi_{s}$. Thin dotted curve in the top pa rels indi ates $\theta_{e} / \theta_{0}$. Dotted/dashed curves between $60^{\circ}$ and $90^{\circ}$ represent with the equilibrium solutions with additional polar cooling/heating.

fr 11 the shear line. The case $Q<0$ may be thought of as a crude representation of radiative cooling in the polar $\mathrm{n}$ gnt, and the case $Q>0$ a representation of latent heat ease from carbon dioxide condensation. The two effects and their interaction with the Hadley cell may therefore be consider $\mathrm{d}$ th through the response to a single forcing term in the temperature equation.

\section{Fül axisymmetric solutions}

\subsection{Convergence to the AMC solutions}

Fo1n wing LH88, we next compare the AMC solutions with steady solutions obtained following the time-evolution of a full axisymmetric model. Whereas LH88 solve the axisymmetric primitive equations, here we take adantage of the relatively simple vertical structure of the solutions and compare instead with an axisymmetric shallow water model. In this formulation, introduced by Held and Phillips
(1990), the meridional component of the shallow water velocity represents the depth average meridional velocity of the Hadley cell. Thermal forcing is represented by a relaxation on the height field to a function $h_{\mathrm{eq}}$ which is exactly equivalent to the depth average $\theta_{e} / \theta_{0}$ of (2), with the mass loss associated with this source term corresponding to a notional vertical velocity out of the layer. Our reasons for comparing with a single-layer representation rather than the full primitive equations are partly numerical efficiency, since in the full eddy resolving calculations of the next section a relatively high resolution is required to capture the eddy dynamics of the annulus instability; and partly because it is desirable to use the simplest model that captures the relevant dynamical processes, here the lateral transport of angular momentum and subsequent instability associated with the horizontal shear. The single-layer representation necessarily neglects details such as the return flow in the boundary layer and allows us to examine the horizontal 
(a) $\mathrm{LH} 88$

$u / a \Omega$

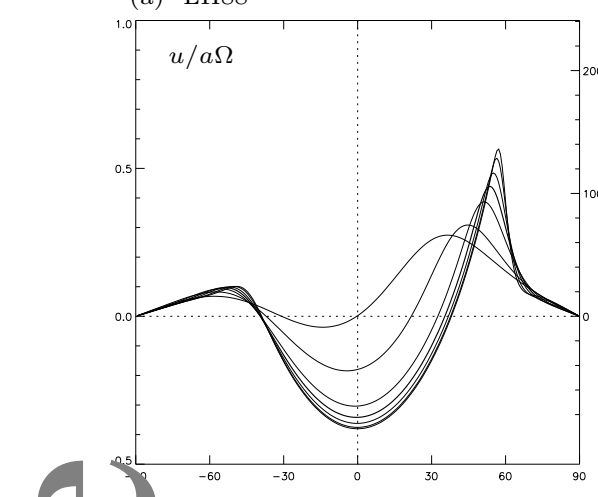

(1)

$q H / \Omega$

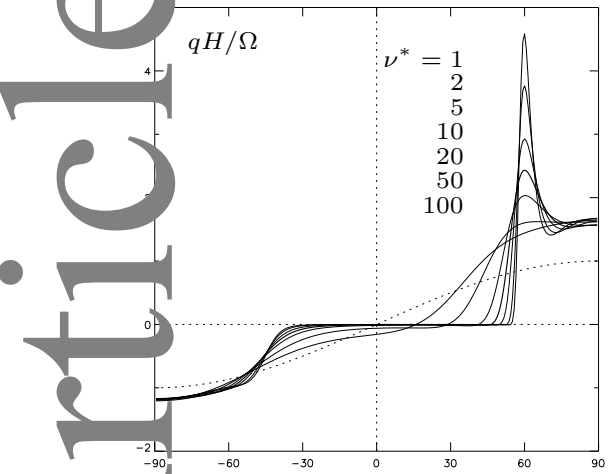

(b) + polar night

(c) + polar night + latent heat
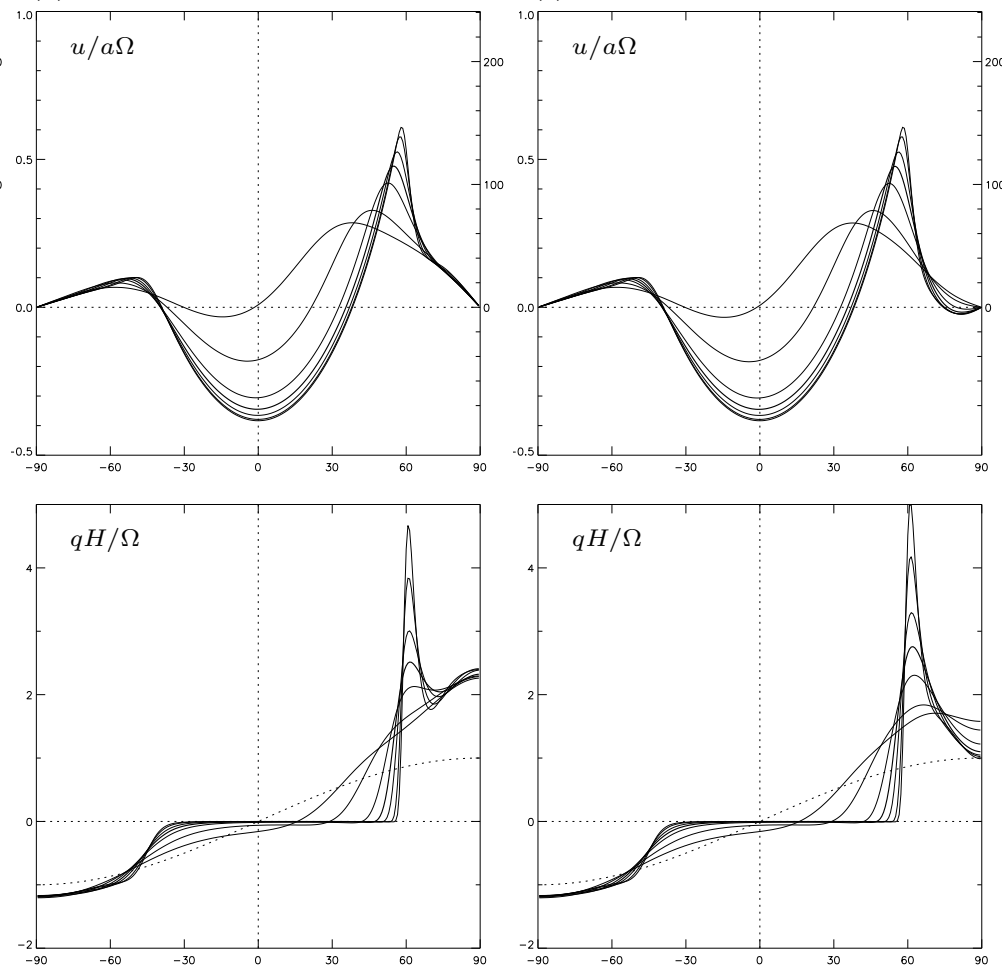

Fio. Jull (axisymmetric) model solutions with parameters as in Fig. 2(b) and diminishing viscosity, $\nu^{*}=\nu N(N+1) \Omega / 2 \pi a^{2}, N=170$, for it $\mathrm{Tere}$ ht choices of $h_{\mathrm{eq}}$ : (a) LH88 profile; (b) LH88 profile with enhanced polar cooling $\left(\phi_{c}=45, Q=-0.1\right.$ ); (c) LH88 profile with enhanced polar coo'sin $\left(\phi_{c}=45, Q=-0.1\right)$ and latent heating $\left(\phi_{c}=60, Q=0.1\right)$. Right-hand ordinate in top panels indicates $\mathrm{m} / \mathrm{s}$.

dynamics without the added complications of baroclinic

\section{in stabilit}

F gurs 3(a) shows the steady state profiles of shallow water zenal velocity $u$ and potential vorticity, $q=\zeta_{a} / h$ ovtained from time integration of the full (axisymmetric) del wi h relaxation to $h_{\mathrm{eq}}$, where $h_{\mathrm{eq}} / H$ is set to the right hand side of (2), and where from now on $H$ is the mean layer depth. T e forcing parameters $\phi_{0}, \Delta$ and Burger number $B$ ar the came as used in Fig. 2(b). A viscosity is required in somentum equations to regularize the delta-function ir $\zeta_{a}$ as ociated with the discontinuity in $u$ of the AMC solution at $\phi_{w}$, with various values of viscous coefficient $\nu$ The discontinuity is captured with increasing Strumness with decreasing $\nu$, and the solutions overall appear to be converging steadily to the AMC solution. As in LH88, the location of maximum wind lies a few degrees equatorward of $\phi_{w}$, though appears to be converging to $\phi_{w}$. In the $\mathrm{AMC}$ region, the zonal velocity is parabolic (in $\sin \phi$ ) and the potential vorticity converges to nearly zero. Near the poles both fields attain thermal wind balance with the equilibrium $h_{\mathrm{eq}}$. It is interesting to note that convergence to the AMC solutions appears to be more robust in the shallow water model than in the primitive equations considered by LH88, another factor in favour of their use here. In particular, the potential vorticity did not appear to approach zero in LH88 and the model became transient below a critical viscosity, a phenomenon argued by Schneider (1984) to be due to non-realizability of the AMC solutions.

Convergence to the AMC solutions gives confidence in the accuracy of the AMC model but the limit $\nu \rightarrow 0$, with its discontinuity in zonal wind, is not particularly realistic. In a full, eddy resolving model, shear instability would be expected to mix potential vortcity and smooth the discontinuity. Viscosity can therefore be considered as a simple representation of horizontal eddy mixing, at least to the extent that eddies mix potential vorticity downgradient Comparison with the eddy-resolving calculations presented in the next section, suggests that a value $\nu=10$, indicated in bold, may be an appropriate choice. With this value of $\nu$, potential vorticity has a local maximum near $60^{\circ}$, about 
$50 \%$ in excess of the polar value, while there is an additional weak local maximum at the pole itself. There is a suggestion of an annular structure but no clear minimum over the pole.

\subsection{Effect of polar heating}

While the LH88 $h_{\text {eq }}$ (or $\theta_{e}$ ) profile provides a simple representation of the seasonal asymmetry in tropical heating, it ly $\mathrm{F}$ artially represents the strong cooling of the winter ponnt. The effect of changing the profile in the polar explicitly include the effect of polar cooling are a st aightt rward consequence of thermal wind balance north of $\varphi_{w}$. Here we add an additional cooling of the form (3) with $\phi_{c}=45^{\circ}$ and $Q=-0.1$, similar to, but slightly b. vaut Inan, the dotted line of Fig. 2(b). The resulting $u$ and $q$ are shown in Fig. 3(b). There is a slight increase in both the zonal velocity and potential vorticity over the ole, including a slight increase in the maximum jet speed, in therm $\mid$ wind balance with the modified $h_{\text {eq }}$, but very little change to the AMC circulation, even although here the extra crofing yoes extend partially into the AMC region.

next an additional perturbation to $h_{\text {eq }}$, of the form (? vith $p_{c}=60^{\circ}$ and $Q=0.1$ as a crude represention of the separate effect of latent heating from carbon dioxide condensation, produces the profiles shown in Fig. 3(c). As w th LH 8 profiles, there is a hollowing out of the core of the yortex, giving a more robust and distributed annular poffle th clear polar minimum, and with the degree of ne -monotonicity controlled directly by the magnitude of th h ng anomaly: strong heating can reduce the polar $\mathrm{p}$, sentia vorticity toward zero or even negative values. Som amount of additional heating, beyond the original $\mathrm{LH}^{\circ}$ profile, was in all cases necessary to change the local ponn maxima in potential vorticity in Fig. 3(a,b) to a local minimum. Again the profiles south of $\phi_{w}$ are comparatively unaffected by the additional heating.

An important point to note is that here both the additional polar cooling $(Q=-0.1)$ and polar heating $(Q=0.1)$ have the same magnitudes and at the pole cancel exactly. The differences in the thermal wind arise because we have chosen the profile of the cooling to be broader $\left(\phi_{c}=\right.$ $\left.45^{\circ}\right)$ than that of the heating $\left(\phi_{c}=60^{\circ}\right)$. The combined perturbation has $d h_{\mathrm{eq}} / d \phi>0$ near the pole, corresponding to a reduced zonal wind and potential vorticity. We acknowledge that our representation of latent heating is grossly oversimplified, but the more compact distribution of latent heating can be partially motivated by its dependence on extreme temperature values, compared with the broader cooling arising from the smooth latitudinal variation of insolation (Toigo et al. 2017).

\subsection{Parameter dependence}

Before proceeding to examine the effect of eddies on the vortex structure, it is interesting to consider briefly what can be deduced from the axisymmetric model in parameter ranges beyond those relevant for Mars. As noted above the LH88 solutions are determined completely by two parameters: the asymmetry $\phi_{0}$ and the combination $R=$ $B \Delta$, where $B=g H / a^{2} \Omega^{2}$ is a planetary Burger number. Planetary parameters such as rotation and stratification therefore affect the solution only by modifying the effective heating amplitude $R$. The dependence of the solution on planetary parameters is essentially the same as that noted recently by Guendelman and Kaspi (2018) in terms of a thermal Rossby number, proportional to $R$.

As seen in Fig. 3, the axisymmetric solutions depend strongly on viscosity. To be useful, therefore, an estimate must be made of appropriate eddy viscosity values, and as noted above comparison with the eddy-resolving calculations presented in the next section, suggests a value $\nu=10$. Using this value, the axisymmetric model provides a crude but quick estimate of possible atmospheric regimes that may arise under different planetary parameters. Fixing the heating and varying $\phi_{0}$, for example, produces the profiles shown in Fig. 4a, which gives an indication of the range of Hadley cell and polar vortex strengths that might have existed on Mars during periods when the planetary obliquity was substantially different from its current value. 
(a) $\Delta=0.15$
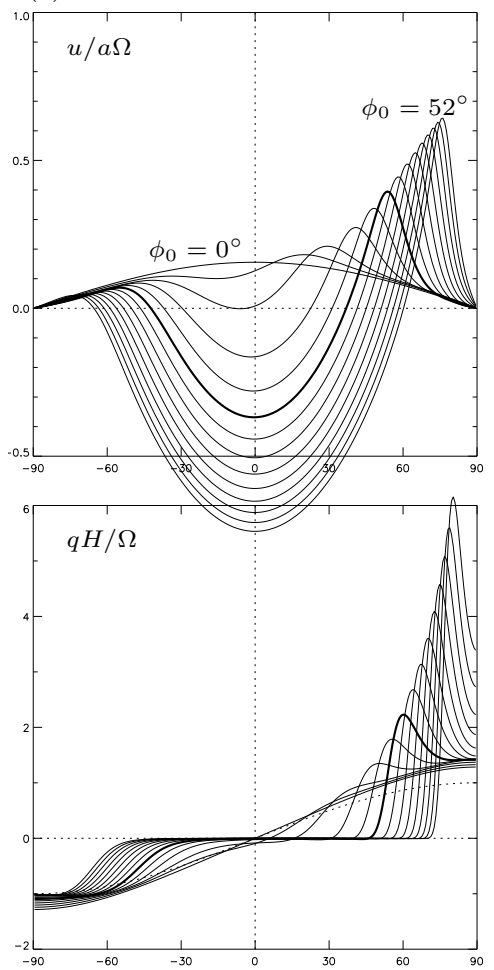

(b) $\phi_{0}=20^{\circ}$
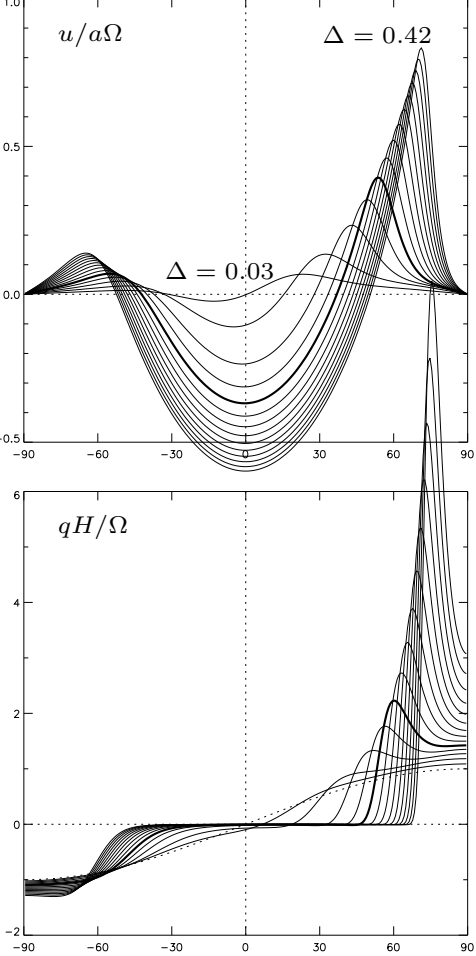

ull (axisymmetric) model solutions with LH88 heating and $\nu=10$. (a) Fixed $\Delta=0.15, \phi_{0}$ ranging from $0^{\circ}$ to $52^{\circ}$ in steps of $4^{\circ}$. (b) Fixed $=20^{\circ}, \Delta$ ranging from 0.03 to 0.42 in steps of 0.03 .

LH88

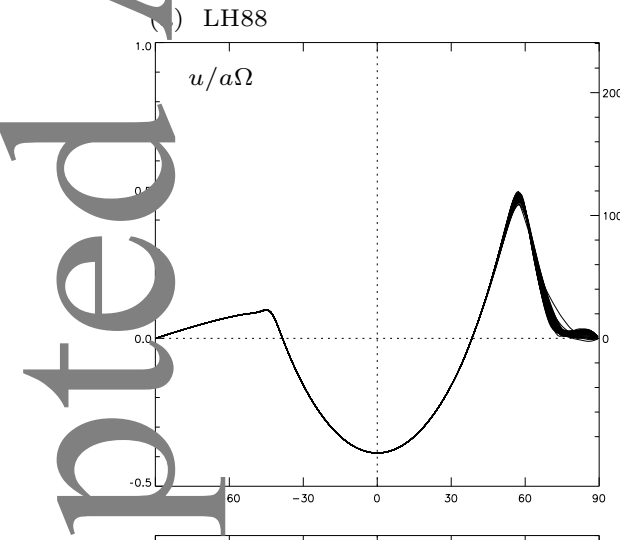

$q H / \Omega$

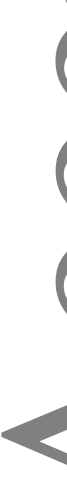

(b) + polar night
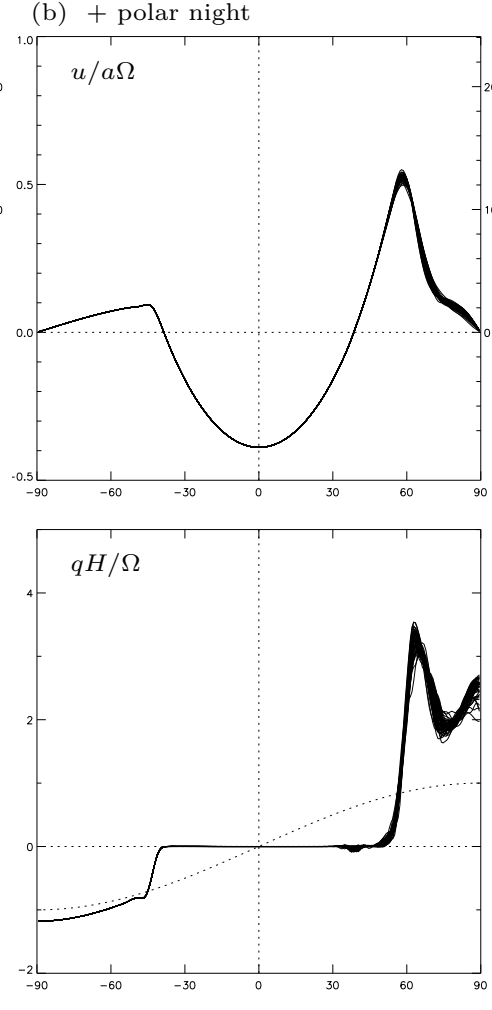

(c) + polar night + latent heat
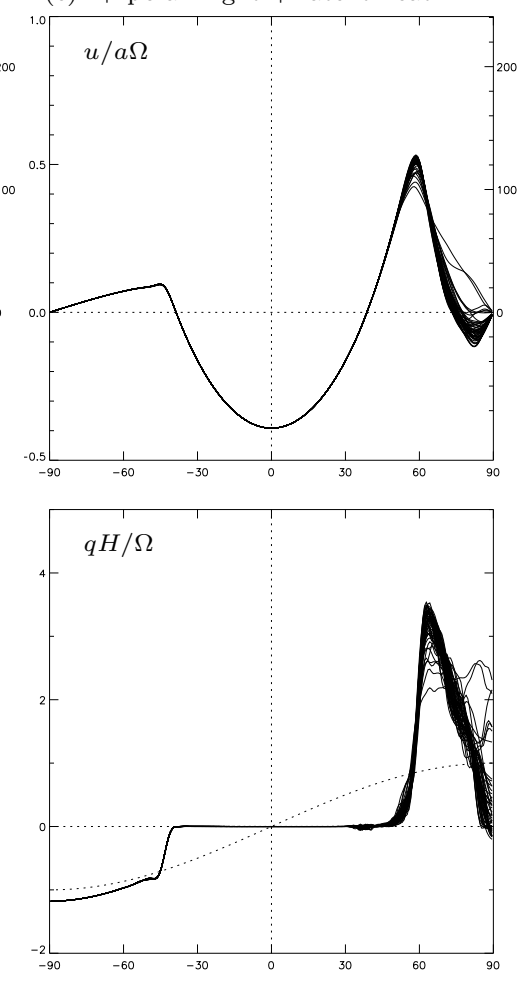

Figure 5. Full eddy resolving model zonal mean fields with parameters as in Fig. 3; lines separated by 10 sol intervals between $t=1500$ and $t=2000$. Units for $u$ : $a \Omega$ (left axis), $\mathrm{ms}^{-1}$ (right axis). Units for $q$ : $2 \Omega$. Equilibrium $h_{\mathrm{eq}}$ : (a) LH88 profile; (b) LH88 profile with enhanced polar cooling $\left(\phi_{c}=45\right.$, $Q=-0.1)$; (c) LH88 profile with enhanced polar cooling $\left(\phi_{c}=45, Q=-0.1\right)$ and latent heating $\left(\phi_{c}=60, Q=0.1\right)$.

Alternatively, for fixed obliquity, the range of possible fixed at the same (Martian) value used above but the same circulations that might be obtained with different heating plot would be obtained by fixing $\Delta$ and varying $B$ such strengths is shown in Fig. $4 \mathrm{~b}$. In these calculations, $B$ is that $R=B \Delta$ varies over the corresponding range. This 
makes explicit how the Hadley cell extent is expected to vary with, say, planetary rotation, all other parameters being equal: lower rotation rate implying larger $B$, hence larger $R$ and a stronger Hadley cell and more poleward polar vortex. Similarly, at a fixed rotation, stronger stratification would again imply a stronger Hadley cell. As pointed out by Guendelman and Kaspi (2018), of the physical parameters that apnear in $B$, rotation rate is the one that shows the lavest ariation in relative value, at least among solar anets.

Jf co urse, come caution is required interpreting such cru stimates; for example, the assumed value of eqay visposity used here is based on the eddy resolving . Delow and may not be the most appropriate one in other regimes, since eddy length scales will also depend $d \mathrm{n} B$ (Walker and Scheider 2006). Nonetheless,

a oove results are consistent with studies in which full generan irculation models have been used to estimate the dependence of Hadley cell structure on different planetary Py sor example, the recent study of Guendelman an $y$ pi (2019) found that Hadley cell width increased w. $1 \mathrm{n}$ inc easing obliquity (increasing $\phi_{0}$ ) and decreasing rotation rate (increasing $B$ ), as in Fig. 4.

\section{Comparison with an eddy resolving model \\ 4. Trodel details}

W nex consider the extent to which the axisymmetric structure obtained above persists in a full, eddy roolvins shallow water model, where the growth of eddres from shear instability is represented. The model eav ons and physical constants are exactly as described in Sovir ur et al. (2017) (equations 1a-1c, therein) but with no topography, $h_{b} \equiv 0$, and solved by the same pseudospectral method with triangular truncation at total wavenumber 170 (T170). We consider the "inviscid limit", in the sense viscosity is set to zero, although the model uses a fourth order hyperdiffusion for numerical stability; however, at all but the smallest scales the hyperdiffusion is much less dissipative than the second order viscosity used above. The equilibrium height $h_{\text {eq }}$ is as used in section 3, namely the LH88 profile with various combinations of additional polar cooling and heating. The relaxation timescale $\tau_{\text {rad }}$ is set to 1 sol in most of the experiments, again following Seviour et al. (2017) and as suggested by Eckermann et al. (2011), with the dependence on this parameter examined in section 4.4 below. A natural extension, not considered here, would be to allow the polar heating to depend interactively on the height field, mimicking the dependence of carbon dioxide condensation on temperature, in a manner similar to that considered recently by Rostami et al. (2018).

\subsection{Influence of polar heating}

Fig. 5 shows the zonal mean zonal velocity and potential vorticity profiles for the same combinations of heating as used in the axisymmetric calculations shown in Fig. 3. Eddies are not explicitly forced in these calculations, but develop from the shear instability associated with the polar potential vorticity distribution. Consequently the flow does not reach a steady state, but does reach a statistical equilibrium on timescales of a few hundred sols. The profiles in Fig. 5 are plotted at 10 sol intervals over the time period $t=1500$ to $t=2000$ sols, which gives an idea of the average profile over that period as well as the degree of transience due to the eddy evolution.

Comparing with Fig. 3, it is seen that the zonal mean evolution states of the full shallow water model are quite similar to the axisymmetric solutions with the value $\nu=$ 10. In particular, in all cases there is a distinct potential vorticity maximum near $60 \mathrm{~N}$ and associated jet maximum, resulting from angular momentum transport from lower latitudes in an AMC circulation. Again, the potential vorticity is nearly exactly zero over most of the AMC region. Poleward of $60 \mathrm{~N}$, the flow is determined by the details of the additional polar cooling or heating. Only with additional heating over the pole is a distinct polar potential vorticity minimum obtained. In all cases, the relatively small spread of individual lines indicates that the zonal mean 
(a) $t=1890$

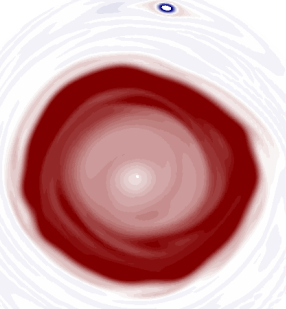

(d) $t=1896$

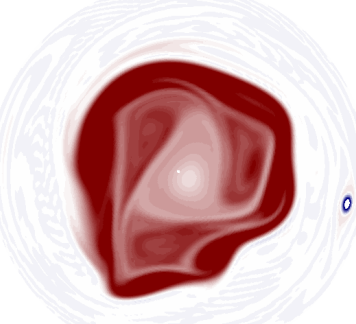

(b) $t=1892$

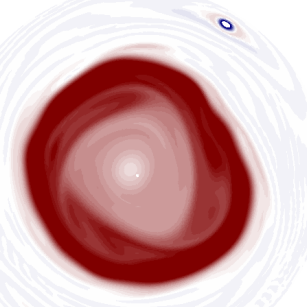

(e) $t=1900$

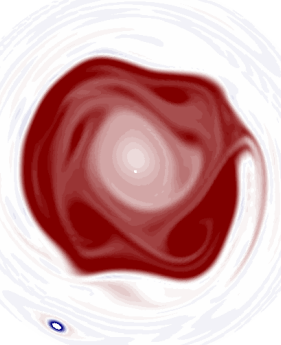

(c) $t=1894$

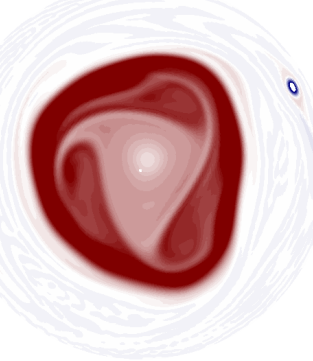

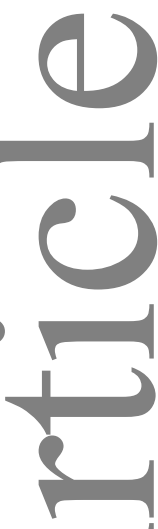

about -40 sols that decreases with increasing $Q$, while ne a nplitude increases. The strong annularity of the vortex in the case $Q=0.1$ shown in Fig. 6c is thus associated with the minimum in $Z$ at $t=2000$ in Fig. 7b. Similarly, the relat vely wavy vortex in the case $Q=0.0$ (Fig. 6a) corresponds to a local maximum in $Z$ at $t=2000$ (Fig. 7a).

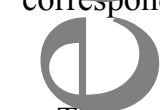

The eyolution of the vortex during the course of a typical vacillation cycle is shown in Fig. 8. The plot shows polar tential vorticity at two and four sol intervals between $t=$ 1890 and $t=1904$, while the corresponding eddy enstrophy can be cen in Fig. 7b. At the beginning of this period thpotor annulus is well formed and almost completely zon-11 oymmetric, with some weak filamentary structure $\mathrm{n}$ ar the nnermost edge remaining from mixing during the previous cycle. Over the next four sols a strong wavenumber thr asturbance grows on the inner edge, and subsequently stron thens to the extent that it causes considerable mixing over a region that gradually encroaches deeper into the annulus core $(t=1900)$. Toward the end of the period shown, most of the eddy enstrophy has been dissipated by strong mixing within the annulus and potential vorticity gradients in the interior are reduced overall. The transience found here is reminiscent of, but on shorter timescales than, vacillation cycles identified in simplified models of the Earth's winter stratospheric polar vortex (e.g. Scott 2016), where the vortex is monopolar, shear stable, and the disturbances are forced through planetary wave excitation from the troposphere below. A closer analogy may be found in the rotating annulus experiments of, for example, Young (2014). The cycle shown in Figure. 7 is typical of the annulus evolution between peaks in the eddy enstrophy.

A more extreme example is shown in Fig. 9 for the case $Q=0.15$ over similarly spaced time intervals between $t=$ 1922 and $t=1936$. Again, a wavenumber three disturbance grows initially on the inner edge, but in this case it grows to large enough amplitudes that the entire annulus is distorted and eventually breaks up completely. Over the next few sols air is mixed vigorously over the entire polar cap, weakening the maximum potential vorticity values. The annular stucture is only gradually recovered from about $t=$ 1940 (not shown).

An examination of potential vorticity maps from both the Mars Analysis Correction Data Assimilation (MACDA v1.0) (Montabone et al. 2014) and Ensemble Mars Atmosphere Reanalysis System (EMARS) (Greybush et al. 2012) reveals a certain amount of variability in the martian 
(a) $t=1922$

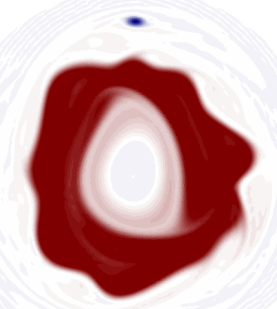

(d) $t=1928$

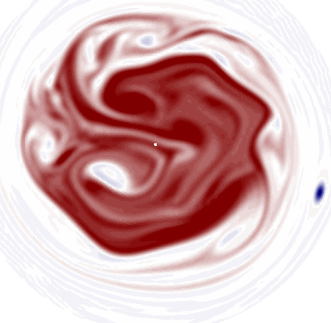

(b) $t=1924$

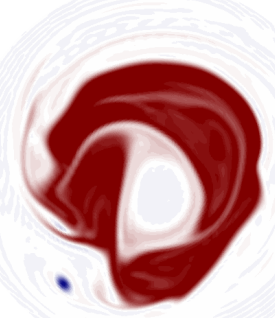

(e) $t=1932$

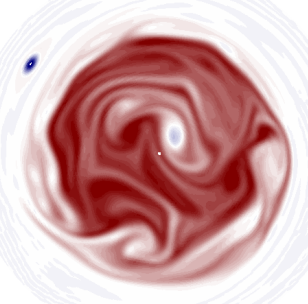

(c) $t=926$

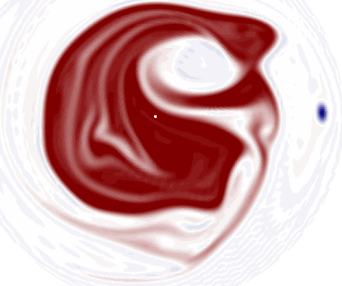

(f) $t=1936$

Figure 9. Potential vorticity for case $Q=0.15$ at times between $t=1922$ and $t=1936$ (a-f).

polar iulus not dissimilar to that presented here (see, g. Naugh et al. (2016), Figure 12 therein). A detailed comparıson is complicated in part by the presence of strong topographic variations on Mars, which provides a Wave forcing to the annulus that is absent in the model integrations. Transience in the observed wavenumber three c.n be ound, as in Fig. 8, but the evolution likely involves interactions between unstable eddy growth and the fixed topographic forcing. The lower resolution of the re nalysi, also makes it harder to follow the Lagrangian evolution of air masses and details of the potential $\mathrm{vart}$ cit mixing. As reanalysis improves we anticipate a $\mathrm{m}$ e ca eful study of transience will be useful, possibly inv $\mathrm{g}$ a decomposition into distinct zonal wavenumbers to separc e transience due to unstable growth from that due to topographic interactions.

completeness, we note that all four cases considered in Fig. 6 exhibit one or two strong compact anticyclones at lower latitudes, around $30 \mathrm{~N}$, in the region of near zero potential vorticity in the AMC region. At first these were thought to be numerical artifacts, possibly arising from the overshoots associated with the hyperdiffusion. Further integrations have been carried out at various resolutions and with different forms of small-scale diffusion, including ordinary viscosity. In all cases, similar anticyclones were obtained and appear to be properly resolved dynamical features. They develop at early times in the model evolution at the onset of the first instability of the shear zone at the edge of the AMC region, and appear to arise from the interaction of the wave breaking with the strong relaxation on the height field. Once formed, they persist, largely independently of the flow at high latitudes. Because they are comparitively small-scale features, their influence on the polar dynamics is presumably weak; their scale in relation to the main polar vortex can be inferred from the small dip in zonal mean profiles in Fig. 5. While interesting, and possibly important for the dyanamics at lower latitudes, they lie beyond the scope of the present work and will not be pursued further here.

\subsection{Influence of Hadley cell}

As discussed in section 2, different combinations of heating asymmetry and heating magnitude can combine to produce the same edge $\phi_{w}$ of the AMC region. The AMC solutions for the three particular choices $\left(\phi_{0}=12, \Delta=0.3\right),\left(\phi_{0}=\right.$ 16, $\Delta=0.3),\left(\phi_{0}=20, \Delta=0.15\right)$ were shown in Fig. 2. We ran the full eddy resolving model with the same 
(a) $Q=0.0$

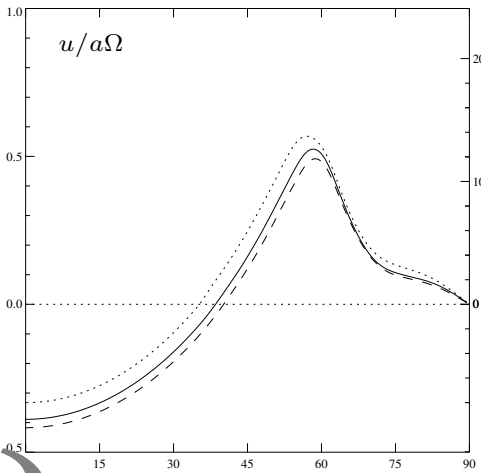

(1)

$q H / \Omega$

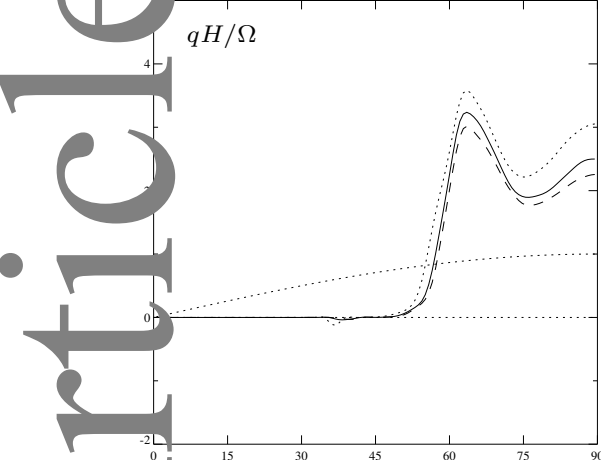

(b) $Q=0.1$
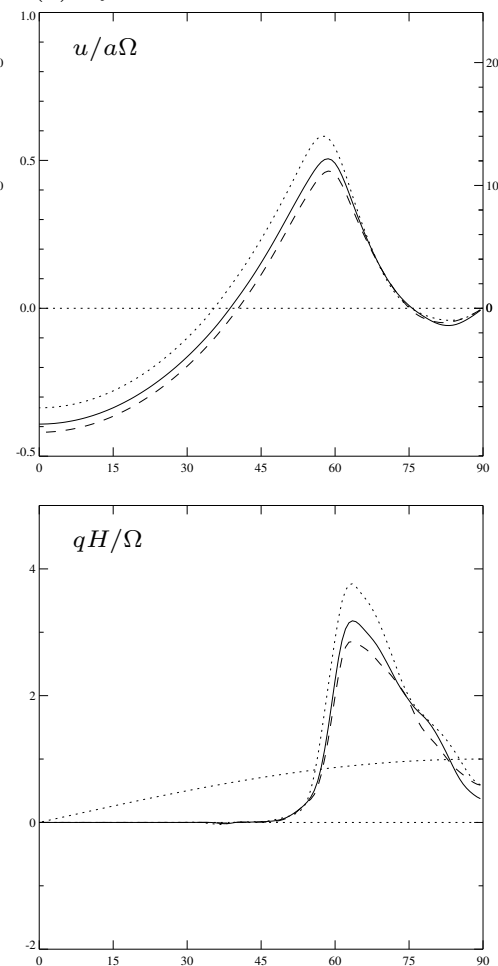

(c) $Q=0.1, \phi_{0}=16$
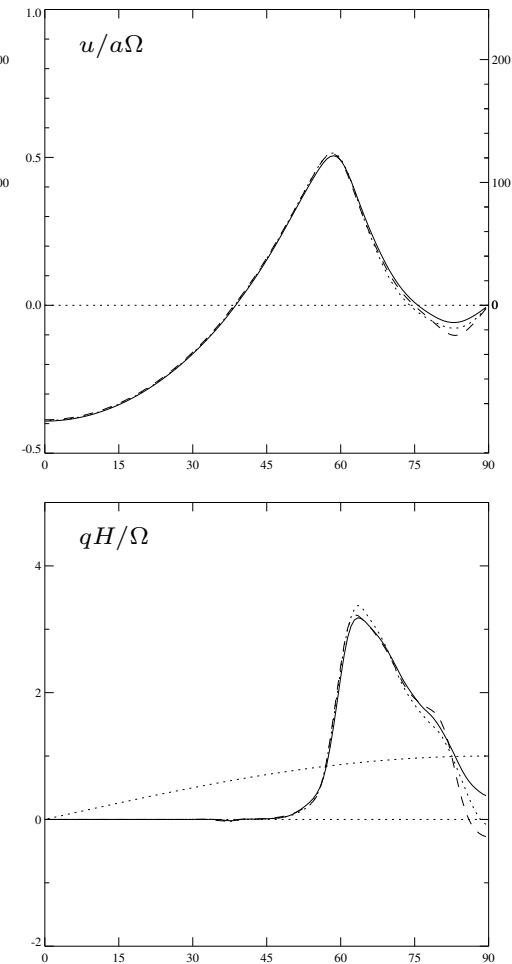

Fig. $\quad$. (a,b) Time-averaged (1500-2000 sol) zonal mean zonal velocity and potential vorticity profiles for cases with Hadley cell forcing $\phi_{0}=12 \mathrm{~S}$, $=1.3$ (dotted), $\phi_{0}=16 \mathrm{~S}, \Delta=0.2$ (solid) and $\phi_{0}=20 \mathrm{~S}, \Delta=0.15$ (dashed): (a) with no latent heating; (b) with latent heating $(Q=0.1$ ). The panal c) shows the corresponding quantities for the case $\phi_{0}=16 \mathrm{~S}, \Delta=0.2$ with latent heating, and for values of $\tau_{\text {rad }}=0.5$ (dotted) and $\tau_{\text {rad }}=2$ (dashed, the case $\tau_{\text {rad }}=1$ (solid) shown again for comparison.

narametclrs, with enhanced polar cooling as above, and with an $d$ witb ut additional polar latent heating. The zonal mean $\mathrm{zO} 1 \mathrm{vo}$ locity and potential vorticity profiles are shown in Fis... 0 , where the profiles have been averaged in time 700 to $t=1000$ for clarity.

The dependence of the AMC flow on $\phi_{0}$ is similar to the solutions snown in Fig. 2, with slightly higher zonal velocity vilues a coss the tropics for $\phi_{0}=12$, decreasing with $\phi_{0}$, regardless of the details of the polar heating. The zonal volocity at the vortex edge shows a similar dependence, as loes the peak potential vorticity. The differences are rela. y small, compared with the two-fold change in the pole-t quator equilibrium height difference across this ronge of $\Delta$.

To measure the extent to which the peak potential vorticity values at $\phi_{w}$ influence the stability of the vortex, we again consider the eddy enstrophy, as defined by (4). For the three cases with polar heating shown in Figure. 10b, we show in Figure 11 the integrated eddy enstrophy as a function of time. the case $\phi_{0}=16$ has been repeated for easy reference.
Eddy enstrophy is largest for the case $\phi_{0}=12$, consistent with the higher peak potential vorticity of the annulus in that case, and decreses with increasing $\phi_{0}$ : time-mean values over the period shown are $0.102,0.072$, and 0.058 respectively. Aside from the decrease in mean values, the variance and characterisic time scales of the variability are broadly similar across the three cases. Clear vacillation cycles occur in all cases, exhibiting somewhat less regularity with increasing $\phi_{0}$, at least over the time interval shown.

\subsection{Influence of radiative timescale}

Finally we consider briefly the effect of $\tau_{\text {rad }}$ on the eddy evolution of the annulus. On Mars, the radiative timescale is short and tightly controls the thermal wind solution poleward of $\phi_{w}$. However, the Hadley cell itself is established on much longer timescales, typically of the order of 500 sols for the cases discussed above. While we expect the radiative timescale to affect the polar dynamics in a manner similar to that discussed in Seviour et al. (2017), such as weaker annular stability at larger $\tau_{\text {rad, }}$ it 


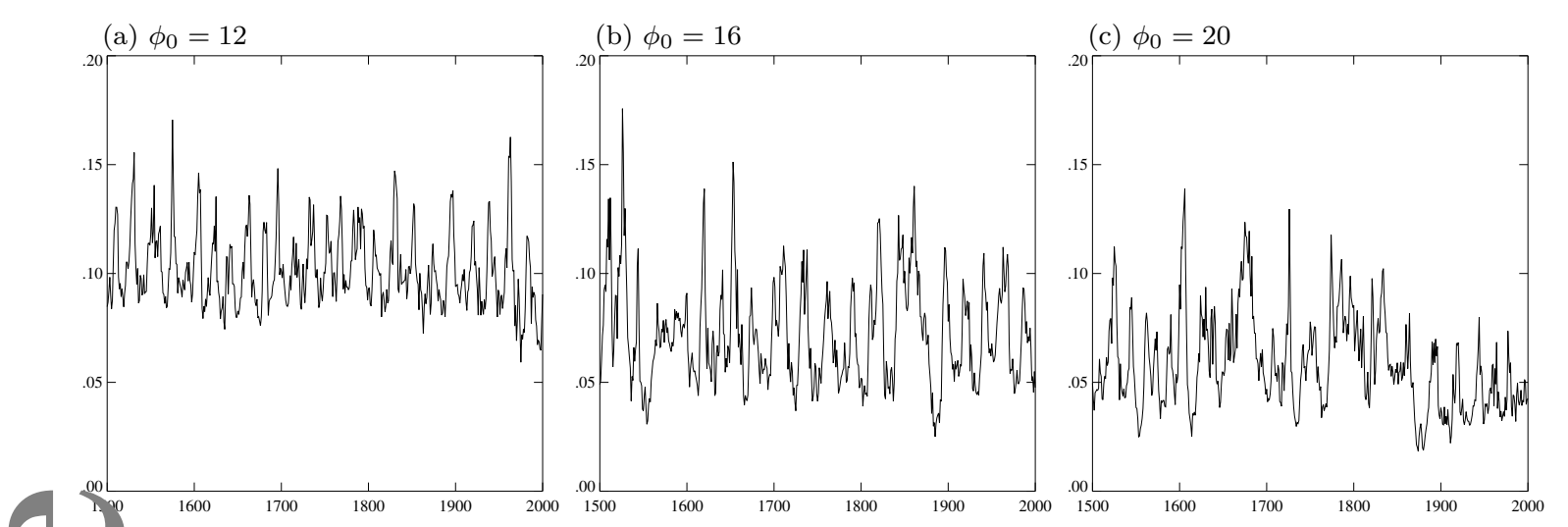

Figure 11. Eddy enstrophy evolution for the three cases shown in Fig. 10b

is nossible that differences may arise due to the way in which $t^{\prime}$ Hadley cell forces the potential vorticity structure or timescales. We consider, therefore, two further simulatis ns in which the value of $\tau_{\text {rad }}=1$ sol used in the p. evious section was increased or decreased by a factor two, keeping all other parameters fixed. *

In e zonal mean zonal velocity and potential vorticity proflios $f$ br the range of $\tau_{\text {rad }}$ are shown in Fig 10c. Both cases $\tau_{\text {rad }}=0.5($ dotted $)$ and $\tau_{\text {rad }}=2($ dashed $)$ are almost identical $\tau_{\text {rad }}=1$ (solid) previously examined, except very clone pole, where there are small variations in the $\mathrm{m}, \mathrm{mul}$ potential vorticity attained. Care has been taken to ensure that the averaging period lies well after the flow hıs reacned a statistically stationary state, in all cases.

The ecdy enstrophy for these simulations are shown in Fig 12 where again the case $\tau_{\text {rad }}=1$ has been repeated for casv eference. $t=1000$ and $t=1100$. Fluctuations in th eda enstrophy again take the form of quasi-regular va $\cdot 11$ on cycles, with characteristic period decreasing with $\tau_{1}$ ad fron about 50 sols at $\tau_{\text {rad }}=2$ to about 20 sols at $\tau_{\text {rad }}=0.5$. In all cases the characteristic period is very short con ared with the time scale for equilibration, but very long com ared with the radiative timescale.

Perhaps surprisingly, the mean eddy enstrophy increases with decreasing $\tau_{\text {rad }}$. It might be expected that the stronger

*Because the representation of latent heating in our model is through a modification of $\theta_{e}$, changing $\tau_{\text {rad }}$ changes the absolute heating associated with this modification. To keep the absolute latent heating fixed would require $\theta_{e}^{\prime}$ to vary inversely with $\tau_{\text {rad }}$, changing the equilibrium state and resulting in a large dependence of the polar state on the $\tau_{\text {rad }}$. Here we are only interested in the effect of $\tau_{\text {rad }}$, keeping $\theta_{e}$ fixed. relaxation over the pole would keep the potential vorticity more tightly constrained in an zonally symmetric annular form. However, it should be remembered that the annular structure here is generated indirectly through the Hadley cell forcing, rather than by direct radiative forcing (again, the main difference between the current formulation and that of Seviour et al. (2017)). Aside from the increase in the mean, the amplitudes of the cycles (peak to peak change) are not strongly dependent on $\tau_{\text {rad }}$.

Other differences in the character of the vacillations can be seen on closer comparison of the $\tau_{\text {rad }}=2$ and $\tau_{\text {rad }}=0.5$ cases. The longer period at $\tau_{\text {rad }}=2$, for example, appears to be associated with a slower increase in eddy enstrophy toward the peak, with a suggestion of a saw-tooth shape in the interval $t=1600-1800$. In these cases the recovery to annular form occurs relatively quickly. Thus it appears the variation in period with $\tau_{\text {rad }}$ is more than a simple linear response to the relaxation timescale. To illustrate the difference, four snapshots of the polar potential vorticity from each of the cases $\tau_{\text {rad }}=2$ and $\tau_{\text {rad }}=0.5$ are shown in Figure 13. For $\tau_{\text {rad }}=2$ (panels a-d, note that panels are at 12 sol intervals), there is a relatively slow increase in wave amplitude from the minimum near $t=1712$ and the maximum near $t=1748$, and a correspondingly gradual increase in the potential vorticity mixing in the annulus. In contrast, for $\tau_{\text {rad }}=0.5$ (panels e- $\mathrm{h}$, and note that panels are now at 2 sol intervals), the distortion of the annulus occurs much faster with strong mixing taking place just 6 sols after the minimum. The more rapid development of the instability 


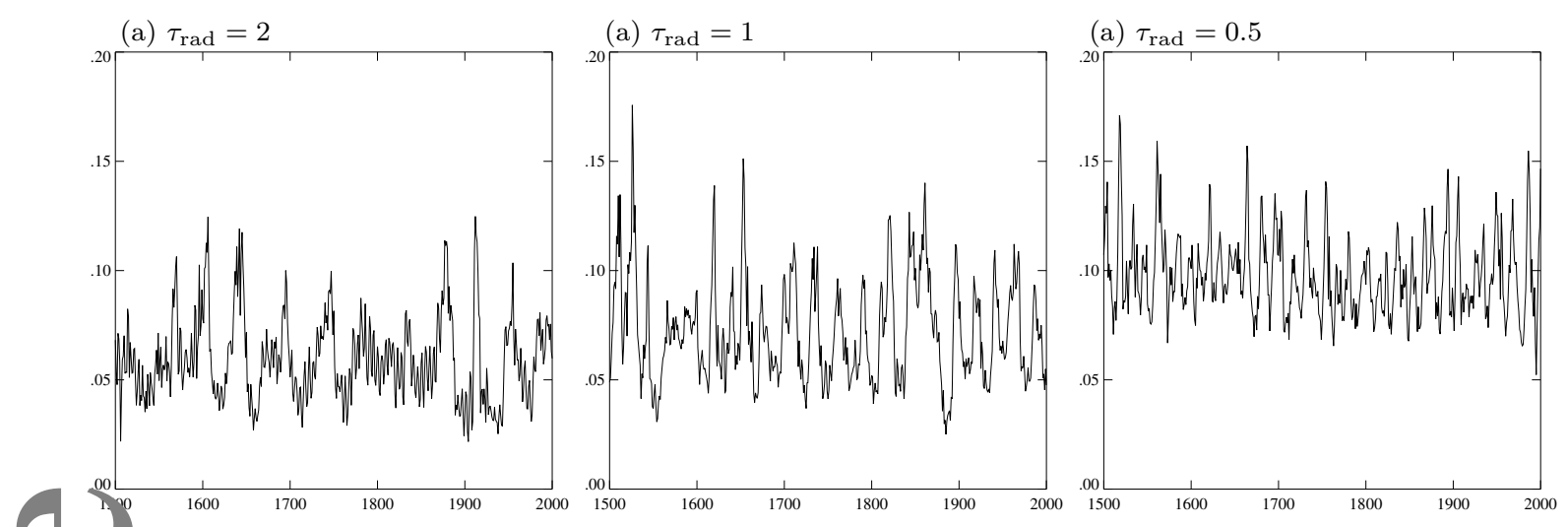

Fis. Eddy enstrophy evolution for the three cases shown in Fig. 10 c: (a) $\tau_{\text {rad }}=2$, (b) $\tau_{\text {rad }}=1$, (c) $\tau_{\text {rad }}=0.5$; in all cases $\phi_{0}=16$ and $Q=0.1$.

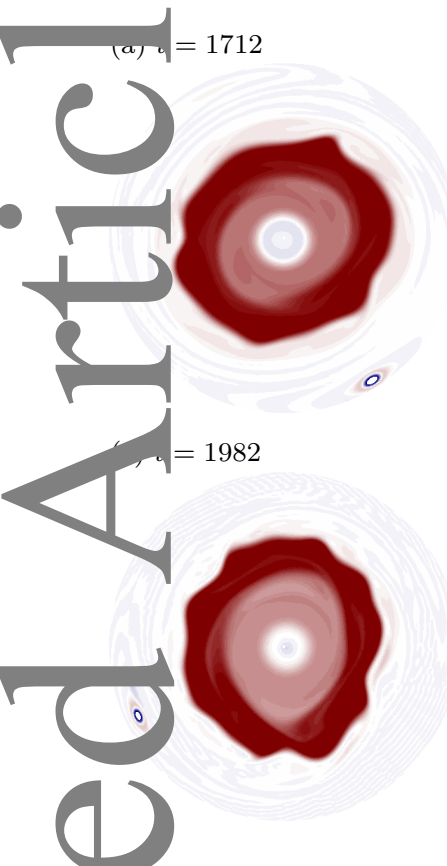

(b) $t=1724$

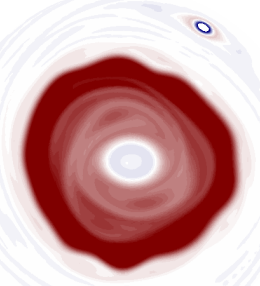

$$
\tau_{\mathrm{rad}}=2
$$

(f) $t=1984$

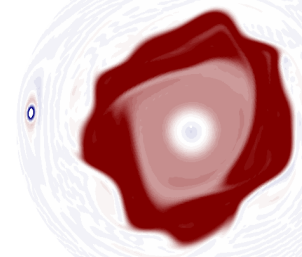

(c) $t=1736$

0

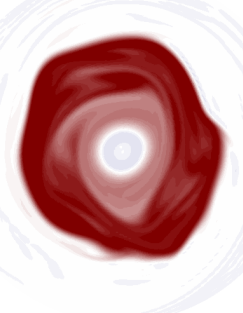

(g) $t=1986$

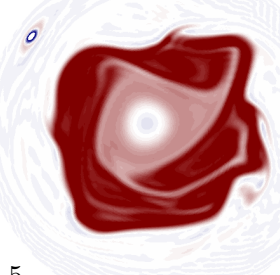

(d) $t=1748$

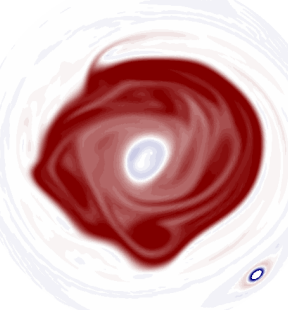

(h) $t=1988$

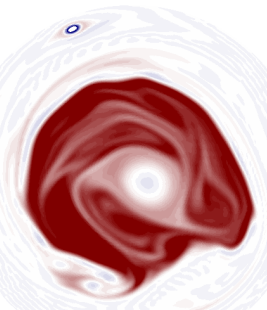

Figure 13. Potential vorticity from simulations with $(\mathrm{a}-\mathrm{d}) \tau_{\mathrm{rad}}=2$, corresponding to Figure. $12 \mathrm{a}$, and $(\mathrm{e}-\mathrm{h}) \tau_{\mathrm{rad}}=0.5$, corresponding to Figure. $12 \mathrm{c}$, epresentative times.

in the latier case cannot be attributed to a direct effect of th shorter radiative timescale, but must arise indirectly thine influence of the zonal mean annular structure o funsta le growth rates. It should be noted, however, that such oenaviour is by no means universal; all three time series e hibit s snificant irregularities, and examples of rapid eddy growth also exist at $\tau_{\text {rad }}=2$ (e.g. $\left.t=1900\right)$.

\section{Con lusions}

We have shown that the annular potential vorticity structure of the Martian polar vortex arises naturally from the combination of two basic forcing mechanisms: an angular momentum conserving Hadley cell driven by offequatorial heating and enhanced heating over the winter pole representing the latent heat release from carbon dioxide condensation. The simplified AMC Hadley cell model of LH88 is capable of capturing the Martian circulation, even though the winter branch of the Hadley cell extends much farther north than on Earth. The model allows the dependence of the polar potential vorticity distribution on each aspect of the forcing to be examined in a clear and unambiguous way. In particular, the location of the zonal wind maximum is controlled mainly by the asymmetry and forcing strength of the Hadley cell, while the details of the flow poleward of the jet are controlled mainly by details of the equilibrium temperature field in that region, through thermal wind balance. 
While the potential vorticity structure of the LH88 model shows no explicit annular structure, the infinite shear at the transition latitude implies a delta-function in vorticity that could lead to a local maximum when smoothed by viscosity or eddy mixing in a full model. However, when the latter effects are included, although a potential vorticity maximum is produced near $60^{\circ} \mathrm{N}$, there is also a weaker but well defined local maximum over the pole. This local polar ximum is stronger when a representation of the cooling of polar night is included. To obtain a di inct yolar minimum on the other hand, some additional hea. is required, the depth of the minimum increasing w GCM experiment of (Toigo et al. 2017) that found a mononblar vortex developed when the parametrization of latent heating from carbon dioxide condensation was ile off, although in our model some degree of the annulan tructure does arise from the Hadley cell alone. The Hadley cell itself imposes a robust constraint on the

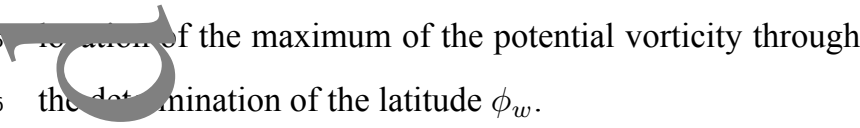

Ir a f 11 , non-axisymmetric shallow water model with resolved eddies the basic dependence on Hadley cell and additional polar heating was confirmed. In this model, with a liative elaxation timescale of $1 \mathrm{sol}$, a stationary state was established on timescales of a few hundred sols, suggesting th at the nal states are attainable on the timescales of the Motian seasonal cycle. Despite the transience associated wis nonlinear eddy evolution arising from shear ir stabilit $y$ of the annulus, the basic annular structure persists in tir - lime mean. Typically, eddy amplitudes were found to wover timescales of a few sols, saturate and then transient mixing of the annular structure. When the eddies are strained to small scales and dissipated the annulus is able to reform under the action of the direct radiative forcing to the thermal wind in the polar regions, giving rise to vacillation cycles on timescales of a few tens of sols. The amplitude of these cycles increases as the additional polar heating is increased, to the point that the vacillations can result in the complete mixing of the annulus across the polar region. Although the vacillation timescale increases with radiative timescale, the dependence does not appear to be linked to a direct change in the restoration timescale of the annulus under radiative forcing, but rather linked to changes in the growth rate of the eddy activity.

Aside from elucidating the mechanisms that control the annular structure, as discussed in section $3 \mathrm{c}$, the axisymmetric model provides a quick means of exploring possible circulation patterns that may exist on other planets. In particular, the AMC model formulation depends explicitly on only two parameters, the latitude of the off-equatorial heating maximum and the effective heating strength. The latter is a combination of the actual pole-to-equator equilibrium temperature difference multiplied by a planetary Burger number that measures the relative importance of rotation and stratification. Thus low rotation or high stratification is associated with higher effective heating strength and a wider Hadley cell. While more detailed aspects of the dynamics will depend on individual physical parameters, the above suggests that when conducting parameter sweep experiments of possible circulation regimes in exo-planetary atmospheres, the effects of solar luminosity, planetary mass, rotation etc, be initially considered in combination.

\section{Appendix}

The latitudes $\phi_{w}, \phi_{s}$, and $\phi_{1}$ in the LH88 model are uniquely determined from the latitude of maximum heating, $\phi_{0}$, by imposing the matching conditions (8)-(11) of LH88. These lead to a system of three equations in the unknowns $\phi_{w}, \phi_{s}$, and $\phi_{1}$, which we provide here for ease of reproduciblity. In terms of a sine-latitude coordinate $y=\sin \phi$, and writing 
$y_{0}=\sin \phi_{0}$ etc, the matching conditions yield the system

$$
\begin{aligned}
& \frac{\left(y_{s}^{2}-y_{1}^{2}\right)^{2}}{1-y_{s}^{2}}-\frac{\left(y_{w}^{2}-y_{1}^{2}\right)^{2}}{1-y_{w}^{2}} \\
& \quad-2 R\left[\left(y_{s}-y_{0}\right)^{2}-\left(y_{w}-y_{0}\right)^{2}\right]=0
\end{aligned}
$$$$
\left(\frac{\left(y_{s}^{2}-y_{1}^{2}\right)^{2}}{1-y_{s}^{2}}-2 R\left(y_{s}-y_{0}\right)^{2}\right)\left(y_{s}-y_{1}\right)
$$$$
-I\left[y_{s}\right]+I\left[y_{1}\right]+\frac{2}{3} R\left[\left(y_{s}-y_{0}\right)^{3}-\left(y_{1}-y_{0}\right)^{3}\right]=0
$$$$
\left(\left(\frac{w}{1-y_{1}^{2}}-2 R\left(y_{w}^{2}-y_{0}\right)^{2}\right)\left(y_{w}-y_{1}\right)\right.
$$

$$
\left.{ }^{L} y_{w}\right]+I\left[y_{1}\right]+\frac{2}{3} R\left[\left(y_{w}-y_{0}\right)^{3}-\left(y_{1}-y_{0}\right)^{3}\right]=0
$$

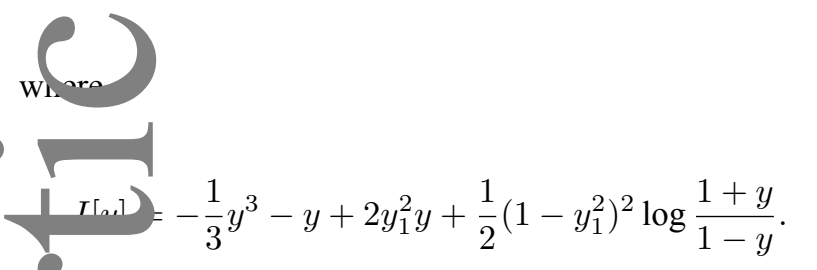

The system can be solved by a suitable iterative scheme Broyden 1965). Notice that the solution $y_{1}, y_{s}, y_{w}$ depends pnly on the latitude of maximum heating $y_{0}$ and the combination of heating strength and planetary Burger

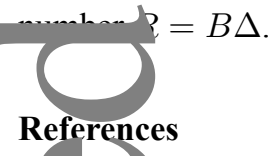

\section{References}

Ban D, Conrath B, Gierasch P, John Wilson R, Smith M. 2004 g waves in the martian atmosphere from MGS TES nadir rarus 170: 365-403.

Barnes JR Haherle RM. 1996. The martian zonal-mean circulation: Angular momentum and potential vorticity structure in GCM si nula ons. J. Atmos. Sci. 53: 3143-3156.

Brovden CG. 1965. A class of methods for solving nonlinear simult eous equations. Mathematics of Computation 19: 577-593.

Dritschel DG, Polvani LM. 1992. The roll-up of vorticity strips on the surface ff a sphere. J. Fluid Mech. 234: 47-69.

Eckermanıl SD, Ma J, Zhu X. 2011. Scale-dependent infrared radiative tping rates on Mars and their role in the deposition of gravity-wave nentum flux. Icarus 211: 429-442.

Greybush SJ, Wilson RJ, Hoffman RN, Hoffman MJ, Miyoshi T, Ide K, McConnochie T, Kalnay E. 2012. Ensemble Kalman filter data assimilation of Thermal Emission Spectrometer temperature retrievals into a Mars GCM. J. Geophys. Res. E 117: 11008.

Guendelman I, Kaspi Y. 2018. An axisymmetric limit for the width of the Hadley cell on planets with large obliquity and long seasonality. Geophys. Res. Lett. 45: 13,213-13,221.
Guendelman I, Kaspi Y. 2019. Atmospheric dynamics on terrestrial planets: the seasonal response to changes in orbital, rotational, and radiative timescales. Astrophys. J. 881: 67.

Held IM, Hou AY. 1980. Nonlinear axially symmetric circulations in a nearly inviscid atmosphere. J Atmos Sci 37: 515-533.

Held IM, Phillips PJ. 1990. A barotropic model of the interaction between the Hadley cell and a Rossby wave. J. Atmos. Sci. 47: 856869.

Hide R. 1969. Dynamics of the atmospheres of the major planets with an appendix on the viscous boundary layer at the rigid bounding surface of an electrically- conducting rotating fluid in the presence of a magnetic field. J. Atmos. Sci. 26: 841-853.

Leovy C. 2001. Weather and climate on mars. Nature 412: 245-249.

Lindzen RS, Hou AY. 1988. Hadley circulations for zonally averaged heating centered off the equator. J. Atmos. Sci. 45: 2416-2427.

McConnochie TH. 2007. Observations of the martian atmosphere: THEMIS-VIS calibration, mesospheric clouds, and the polar vortex. Ph.D. thesis Cornell University: https://ecommons.cornell.edu/handle/1813/3517.

Mitchell DM, Montabone L, Thomson S, Read PL. 2015. Polar vortices on Earth and Mars: A comparative study of the climatology and variability from reanalyses. Q. J. Roy. Meteor. Soc. 141: 550-562.

Montabone L, Lewis S, Read P, Hinson D. 2006. Validation of martian meteorological data assimilation for MGS/TES using radio occultation measurements. Icarus 185: 113-132.

Montabone L, Marsh K, Lewis SR, Read PL, Smith MD, Holmes J, Spiga A, Lowe D, Pamment A. 2014. The Mars Analysis Correction Data Assimilation (MACDA) dataset v1.0. Geosci. Data J. 1: 129-139.

Richardson MI, Wilson RJ. 2002. A topographically forced asymmetry in the Martian circulation and climate. Nature 416: 298-301.

Rostami M, Zeitlin V, Montabone L. 2018. On the role of spatially inhomogeneous diabatic effects upon the evolution of Mars' annular polar vortex. Icarus 314: 376-388.

Schneider EK. 1983. Martian great dust storms: Interpretive axially symmetric models. Icarus 55: 302-331.

Schneider EK. 1984. Response of the annual and zonal mean winds and temperatures to variations in the heat and momentum sources. J. Atmos. Sci. 41: 1093-1115.

Scott RK. 2016. A new class of vacillations of the stratospheric polar vortex. Q. J. Roy. Meteor. Soc. 142: 1948-1957.

Seviour WJM, Waugh DW, Scott RK. 2017. The stability of Mars' annular polar vortex. J. Atmos. Sci. 75: 1533-1547.

Toigo AD, Waugh DW, Guzewich SD. 2017. What causes Mars' annular polar vortices? Geophys. Res. Lett. 44: 71-78. 
